\title{
Limitations of Applicability of the Green-Kubo Approach for Calculating the Thermal Conductivity of a Confined Liquid in Computer Simulations
}

\author{
Krzysztof Hyżorek ${ }^{1}$, Konstantin V. Tretiakov ${ }^{1,2, *}$ \\ ${ }^{1}$ Institute of Molecular Physics \\ Polish Academy of Sciences, Smoluchowskiego 17, 60-179 Poznań, Poland \\ ${ }^{2}$ President St. Wojciechowski PWSZ in Kalisz. \\ Nowy Swiat 4, 62-800 Kalisz, Poland \\ *E-mail: kvt@ifmpan.poznan.pl
}

Received: 18 November 2016; revised: 24 November 2016; accepted: 25 November 2016; published online: 06 December 2016

\begin{abstract}
Thermal conductivity $(\lambda)$ of the Lennard-Jones liquid in cylindrical nanochannels has been determined using the Green-Kubo (GK) approach in equilibrium Molecular Dynamics simulations. Good convergence of $\lambda(\tau)$ has been observed along the nanochannel's axis where the periodic boundary conditions are applied. However, it has been found that the estimation of limiting value of $\lambda(\tau)$ in the transverse direction, where walls confine the liquid, is ambiguous.
\end{abstract}

Key words: the Green-Kubo method, thermal conductivity, simple liquids, nanochannels, Molecular Dynamics simulations

\section{INTRODUCTION}

Recent developments in nanoscience and nanotechnology [1] have caused increasing interest in fundamental knowledge regarding behavior of systems with strong geometrical confinements. In this context, studies of simple liquids in nanochannels can lead to better understanding of the influence of a strong geometrical confinement on physical properties, e.g., on heat transfer (which is characterized by thermal conductivity). Unfortunately, theoretical methods allowing to describe such systems are poorly developed, which seriously impedes scientific progress.

Rigorous derivation of transport coefficients (including thermal conductivity) in bulk limit has been performed by Kubo and Zwanzig in their now famous papers [2,3]. Petravic and Harrowell generalized the Green-Kubo (GK) formalism describing the transport between two arbitrarily located parallel planes within a sample [4-6]. Despite the fact that there is no theoretical basis for using the original GK method for con- fined systems, in literature one can find works whose authors use the GK formalism (due to its simplicity) for determining thermal conductivity in the case of nanoslits $[7,8]$ and nanochannels [9]. It is worth adding that the application of this method to systems with geometrical confinement can be interesting at least for two reasons. Firstly, this will allow to check whether this method can be applied in a wider range than it was originally derived for. Secondly, this study may inspire theorists to generalize this method for confined systems. In this context, in the present paper we consider some practical aspects of applying the GK method to calculate thermal conductivity of a confined system by using computer simulations.

The paper is organized as follows. In Sec. II the model of the studied system, the method used to calculate thermal conductivity, and details of the simulations are presented. Applicability of the GK method for calculation of thermal conductivity of liquid in a circular nanochannel is discussed in Sec. III. Conclusions are drawn in Sec. IV. 


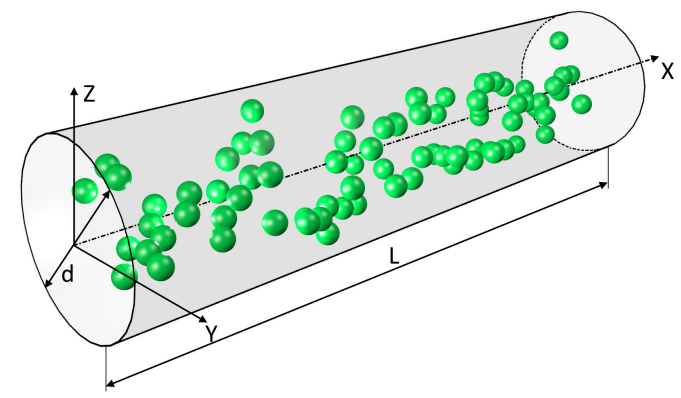

Fig. 1. Schematic representation of the studied cylindrical channel. $L$ is the length of the channel. The periodic boundary conditions have been applied in $x$-direction. $d$ is the diameter of the channel.

\section{PRELIMINARIES}

\section{1. Model}

In this study we are considering a cylindrical nanochannel filled with a liquid. Geometry of the studied system is presented in Fig 1. The particles representing the liquid interact via Lennard-Jones (LJ) interatomic, pair-wise potential [10]

$$
\phi\left(r_{i j}\right)=4 \epsilon\left[\left(\frac{\sigma}{r_{i j}}\right)^{12}-\left(\frac{\sigma}{r_{i j}}\right)^{6}\right]
$$

where $\epsilon$ defines the energy scale, $\sigma$ is the particle diameter and $r_{i j}$ is the distance between atoms $i$ and $j$. The particlewall interaction is set by the steep, repulsive, inverse-power potential

$$
V^{\text {wall }}(r)=\epsilon\left(\frac{\sigma}{r}\right)^{96},
$$

where $r$ is the distance between the wall and a particle. The main reason behind using this kind of potential is to avoid difficulties in determining the volume of the channel, which is necessary to calculate thermal conductivity. In this case the channel volume is exactly the same as the one available for the particles [9]. In addition, a steep, repulsive interaction allows to minimize wall's influence on the particles representing the liquid.

\section{2. Method}

Thermal conductivity $(\lambda)$ is described by the Fourier's law

$$
\vec{J}=-\lambda \nabla T,
$$

where $J$ is the heat current and $T$ is temperature ( $\lambda$ is a tensor, in general). In the considered system, thermal conductivity along the channel ( $x$-direction) and across the channel ( $y, z$ directions) is different due to geometry of the studied system.

Thermal conductivity in $\gamma$-direction, where $\gamma$ represents the $x$-direction (along channel) or the transverse direction (across channel) has been calculated using the Green-Kubo formula $[2,3,11]$ :

$$
\lambda_{\gamma}=\frac{V}{k_{B} T^{2}} \int_{0}^{\tau} d t<j_{\gamma}(0) j_{\gamma}(t)>,
$$

where $k_{B}$ is the Boltzmann constant and $V$ is the volume. The microscopic heat current $j$ [12] is given by equation $[3,10,13]$

$$
\boldsymbol{j}(t)=\frac{1}{V}\left(\sum_{i} \boldsymbol{v}_{i} \varepsilon_{i}+\frac{1}{2} \sum_{i} \sum_{j, i \neq j} \boldsymbol{r}_{i j}\left(\boldsymbol{v}_{i} \cdot \boldsymbol{F}_{i j}\right)\right),
$$

where $\boldsymbol{v}_{i}$ is the velocity of particle $i, \boldsymbol{F}_{i j}$ is the force on atom $i$ due to atom $j$. The microscopic site energy $\varepsilon$ is defined by equation

$$
\varepsilon_{i}=\frac{1}{2} m_{i}\left|\boldsymbol{v}_{i}\right|^{2}+\frac{1}{2} \sum_{i} \phi\left(\boldsymbol{r}_{i j}\right)
$$

where $m$ is the mass of particle. One can find more details concerning the method in Refs. [13, 14].

\section{3. Computational details}

In order to determine thermal conductivity the equilibrium molecular dynamics (EMD) simulations have been performed. The Verlet velocity integration algorithm was used to integrate the equations of motion. To control the temperature the velocity scaling scheme was applied. Initial configurations of particles in the channel have been created using the procedure from Ref. [9]. The size of the nanochannel in the $x$-direction was $x=11.29 \sigma$. Sizes in the transverse direction were equal to: $8.66 \sigma, 10,50 \sigma$, and $12.24 \sigma$. The number of particles for those systems were $N=312, N=480$, and $N=672$, respectively. The cut-off radius of the LJ potential was set to the half of the system width, except for the largest channel where it was set to $5 \sigma$. In the case of the particle-wall interaction potential no cut-off radius was applied. Periodic boundary conditions were applied in the $x$-direction. The integration time step was set to $\Delta t=0.001 \tau\left(\tau=\sqrt{m \sigma^{2} \epsilon}\right)$. MD simulations consisted of $4 \times 10^{5}$ time steps with additional $10^{5}$ time steps to equilibrate the systems. All simulations were performed for density $\rho^{*}=N / V=0.6$ at temperature $T^{*}=k_{B} T / \epsilon=1.4$. Thermal conductivity coefficients $\left(\lambda^{*}=\lambda T \sigma \tau / k_{B} \epsilon\right)$ were averaged over 10 independent runs.

\section{RESULTS AND DISCISSION}

It is well known that the GK method was derived for a homogeneous system in the bulk limit $[2,3]$. On the other hand, it is known that the liquid close to the walls is not homogeneous [11]. In Fig. 2, one can find the strong structuring of liquid near the wall of the channel. However, the density 
profile in Fig. 2 shows that even in the case of a narrow channel the structure of the liquid vanishes in the middle of the channel where the liquid can be considered to be homogeneous. Therefore, in practice, one can neglect the structure effects close to the walls and use the average density. This assumption is at least reasonable for channels with large crosssections if the measurement is performed in the $x$-direction (along the channel) where periodical boundary conditions are applied. Recently, it has been shown that the value of thermal conductivity along a channel obtained by the GK method is in good agreement with the results obtained by the NEMD [9]. This can suggest applicability of the GK method for confined systems in direction where the periodic boundary conditions are applied, i.e., in the direction without any confinement.

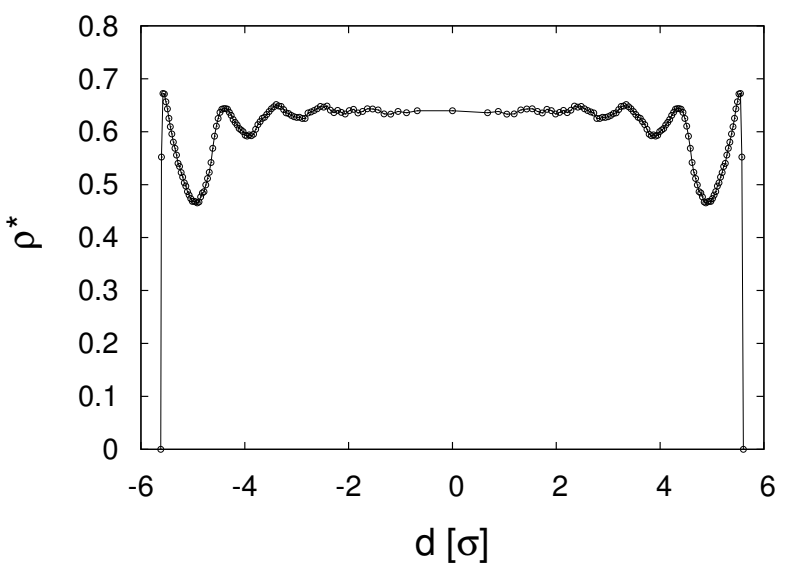

Fig. 2. The density profiles for channel with diameter equal to $12.24 \sigma$.

In the present study, apart from thermal conductivity along a channel, we have also calculated the heat current autocorrelation functions for direction perpendicular to the channel's axis. The obtained results, after direct application of Eq. 4 with limiting time $\tau$, are shown in Fig. 3. As one can see, along the channel (in the $x$-direction) where the periodic boundary conditions are applied the quantity $\lambda(\tau)$ converges to a limiting value as it was reported previously [9]. However, in the transverse direction (across the channel) the situation is quite different. The quantity $\lambda(\tau)$, after initial increase, decreases significantly with $\tau$ in such a way that the estimation of limiting value (if exists) is ambiguous and problematic. In literature [8], one can find attempts to use the GK formalism in direction of confinement but those results are probably obtained by considering too short time $\tau$ for the integral (Eq. 4) and using a local plateau as a limiting value (as e.g., $2<\tau<4$, see the full symbols in Fig. 3). From this it can be concluded that it is not correct to use equation (4) for confined systems in the transverse direction in which confinement is applied and hence the periodic boundary conditions cannot be used. The Green-Kubo formalism is only valid in the limit of zero surface to volume ratio in the present context.

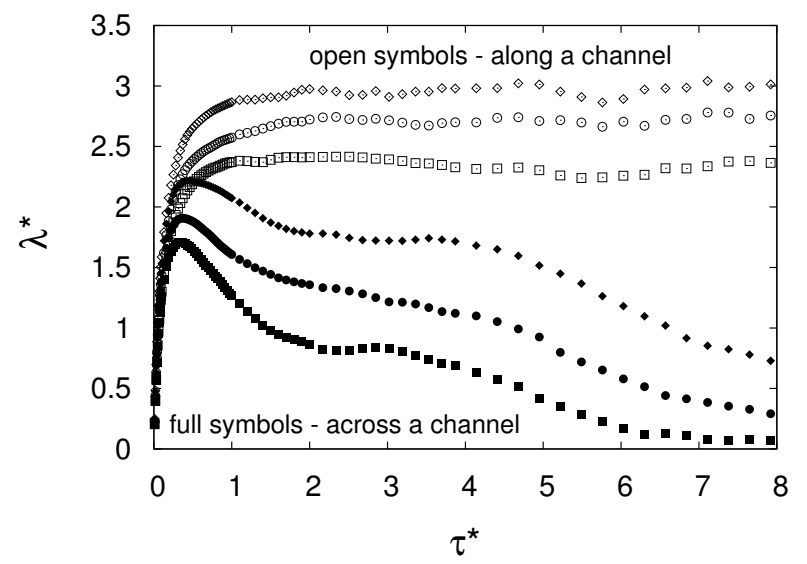

Fig. 3. Integral (eq. 4) for calculation of thermal conductivity as a function of time $\left(\tau^{*}=\tau / \sqrt{m \sigma^{2} \epsilon}\right)$ for three nanochannels of diameters: $8.66 \sigma$ (squares), $10.50 \sigma$ (circles) and $12.24 \sigma$ (diamonds).

\section{CONCLUSIONS}

Our research suggests that despite the lack of theoretical background, the Green-Kubo formalism can be applied to confined systems when certain assumptions are satisfied.

In sum, the Green-Kubo formula can be used for calculating thermal conductivity if the structure effects close to the walls can be considered as an inessential perturbation. This assumption is reasonable along the channel in the $x$ direction where the periodic boundary conditions are applied (i.e., in the direction without any confinement) at least for large cross-sections of a channel. Whereas in the transverse direction (across the channel) the above conditions are not satisfied and as a result $\lambda(\tau)$ does not converge to a limiting value. Therefore, in practice, thermal conductivity across the channel cannot be calculated using original Green-Kubo formula.

\section{Acknowledgements}

This work was supported by the Polish National Science Center grants DEC-2012/05/B/ST3/03255. Part of the calculations were performed at the Poznan Computing and Networking Center (PCSS).

\section{References}

[1] M. Bacon, S.J. Bradley, T. Nann, Graphene Quantum Dots, Part. Part. Syst. Char. 31(4), 415 (2014).

[2] R. Kubo, J. Phys. Soc. Japan 12 (1957).

[3] R. Zwanzig, Time-Correlation Functions and Transport Coefficients in Statistical Mechanics, Annu. Rev. Phys. Chem., 16 (1965).

[4] J. Petravic, P. Harrowell, Linear response theory for thermal conductivity and viscosity in therms of boundary fluctuations, Phys. Rew. E 71(061201) (2005). 
[5] J. Petravic, P. Harrowell, An equilibrium calculation of thermal transport coefficients between two planes of arbitrary separation in a condensed phase, J. Chem. Phys. 124, 0445112 (2006).

[6] J. Petravic, P. Harrowell, Equilibrium calculations of viscosity and thermal conductivity across a solid-liquid interface using boundary fluctuations, J. Chem. Phys. 128(194710) (2008).

[7] A.E. Giannakopoulos, F. Sofos, T.E. Karakasidis, A. Liakopoulos, Unified description of size effects of transport properties of liquids flowing in nanochannels, Int. J. Heat Mass Tran. (55), 5087-5092 (2012).

[8] M. Frank, D. Drikakis, N. Asproulis, Thermal conductivity of nanofluid in nanochannels, Microfluid. Nanofluid. 19(5), 1011-1017 (2015).

[9] K. Hyżorek, K.V. Tretiakov, Thermal conductivity of liquid argon in nanochannels from molecular dynamics simulations, J. Chem. Phys. 144, 194507 (2016).
[10] M. P. Allen, D. J. Tildesley, Computer Simulation of Liquids, J. W. Arrowsmith Ltd., Bristol, UK, 1987.

[11] J.P. Hansen, I.R. McDonald, Theory of Simple Liquids, Academic, New York, 2005.

[12] J. G. Kirkwood, The Statistical Mechanical Theory of Transport Processes I. General Theory, J. Chem. Phys. 14 (1946).

[13] D. Heyes, Transport-Coefficients of Lennard-Jones Fluids - A Molecular-Dynamics and Effective-Hard-Sphere Treatment, Phys. Rev. B 37, 5677 (1988).

[14] K.V. Tretiakov, S. Scandolo, Thermal conductivity of solid argon for molecular dynamics simulations, J. Chem. Phys. 120(8), 3765-3769 (2004).

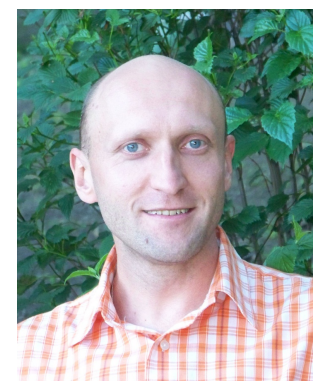

Konstantin V. Tretiakov graduated summa cum laude in industrial electronics from the The Pavel Sukhoi State Technical University (Gomel, Belarus) in 1994. He received his PhD degree in Physics from the Institute of Molecular Physics, Polish Academy of Sciences (Poznan, Poland) in 2000. In 2002-2004, Dr. Tretiakov was a Postdoctoral Fellow (with Prof. S. Scandolo) in the Condensed Matter and Statististical Physics Section at the Abdus Salam International Center for Theoretical Physics (Trieste, Italy). In 2007-2009, Dr. Tretiakov worked with Prof. B. Grzybowski in the Department of Chemical and Biological Engineering at Northwestern University (Evanston IL, USA). Since 2014 he is Professor IFM PAN in Department of Computational Physics of Complex Systems headed by Professor Wojciechowski. Dr. habil. Tretiakov specializes in statistical physics and computer simulation of many body systems, with research focusing on non-equilibrium self-assembling systems, transport properties of soft-matter, and elastic properties of solids, in particular materials exhibiting negative Poisson's ratio.

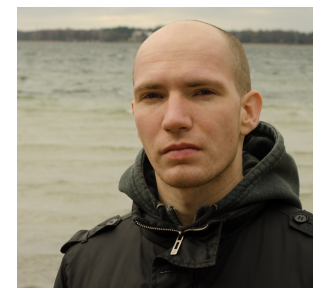

Krzysztof Hyżorek graduated in Technical Physics in 2014 from Poznan University of Technology. He specializes in computer simulations, focusing his interest on transport properties of simple fluids in confined systems. 